\title{
Thermodynamics of the planetary transitions from barren worlds to habitability and advanced technological societies
}

\author{
DR. AXEL KLEIDON ${ }^{1}$, ADAM FRANK ${ }^{2}$ AND MARINA \\ ALBERTI $^{3}$ \\ ${ }^{1}$ Max Planck Institute for Biogeochemistry \\ ${ }^{2}$ University of Rochester \\ ${ }^{3}$ University of Washington \\ Presenting Author: akleidon@bgc-jena.mpg.de
}

Planetary dynamics result from work being done: acceleration maintains motion, lifting and dehumidification maintains hydrologic cycling within the atmosphere, photosynthesis fuels metabolisms of complex life, and power plants fuel industrialized societies. Hence, planetary environments can be distinguished by which types of work-performing processes these sustain, how much free energy these generate, and which forms of disequilibrium these maintain [1]. Here, we extend this perspective and ask about the necessary conditions, the dominant dynamics, and about the key feedbacks that allow lifeless worlds to become habitable, evolve complex life, and transitions into an Anthropocene state. The foundation is set by thermodynamics as it sets directions and limits the magnitude of free energy generation. Heating differences can generate mechanical work, driving planetary motions and hydrologic cycling. Photochemistry can, in principle, generate free energy out of sunlight more efficiently, but it needs an environment that sustains motion to separate backward and forward reactions. When combined, this can sustain high levels of metabolic activity, such as those found in tropical rainforests on Earth. Metabolic activity transforms the geochemical environment, feeding back to the conditions that sustain motion. This can make the environment more habitable, sustain higher metabolic activities, evolve towards greater complexity, forming a positive feedback on habitability. Human activity draws from the free energy generated by life for its metabolic needs and for fuelling its technosphere, thereby introducing a new level of processes that alter planetary dynamics. The work and transformations done by human activity alter the planetary environment, feeding back to either degrading or improving the planetary conditions to generate free energy. We illustrate this approach with a simple model that describes the dynamics of free energy similar to population dynamics in biology, but add feedbacks that deteriorate or enhance the capacity to generate energy from the planetary forcing. We suggest that by viewing the whole Earth as an energy-converting planet provides a unifying perspective that quantifies the substantial role of human activity, but which also points out the conditions needed to sustainably evolve the planet to a state of greater activity.

[1] Frank, Kleidon, Alberti (2017) Anthropocene 19: 13-21. doi:10.1016/j.ancene.2017.08.002. 